\title{
Digital Control System of a DC Motor Using a Proportional-Integral-Derivative (PID) Controller
}

\author{
Aaron Don M. Africa, Darlene Alyssa P. Abaluna, Ara Jyllian A. Abello, Joaquin Miguel B. Lalusin \\ Department of Electronics and Communications Engineering \\ De La Salle University, Manila \\ 2401 Taft Ave., Malate, Manila 1004, \\ Philippines, aaron.africa@dlsu.edu.ph
}

\begin{abstract}
Proportional-Integral-Derivative (PID) Controllers are some of the most widely used control systems used in the industry. Coupled with the fact that DC motors are the most commonly used actuators in its class, the combination of the two merits a look into how the performance of the whole system will look like. In this paper, the objective is to develop and tune a system with a DC motor, a closed-loop control system, and an integrated PID controller. A setpoint speed will be the target for the motor reach with feedback sensors and an introduced noise in the control system, the PID controller must maintain the speed of the motor at or near the setpoint target. Another objective is to analyze and decide how the control system should be tuned. The whole system will be modeled using Simulink and the Control Systems Toolbox which are both available in MATLAB.
\end{abstract}

Key words: dc motor, control system, PID controller, Simulink.

\section{INTRODUCTION}

DC motors, dated back in the 1900s, have persisted in modern technology due to its major contributions in powering various industrial applications. A DC motor, as the name implies, is powered by a direct current. The objective of this device is to convert electrical energy into mechanical energy. This has given people in the 19th century the convenience to drive a boat using these motors, and the exact same convenience to the essential appliances in the present time. It is commonly utilized in the field of transportation, mining, defense, and construction $[1,2,3,4]$.

As DC quickly overtook the AC, DC motors started to become widely used in the industries; hence, controlling such devices has sparked interest to a vast number of researchers and industries. There are two important characteristics of motors: torque and speed; where the ability of the motor to do work is derived from the speed and the torque.

Methods and approaches on how to control these motors differ in complexity. Some modern control techniques such as optimal control, nonlinear control,adaptive control, and variable structure controlwere proposed to control the speed of a magnet DC motor that is brushless and permanentbut these tend to be complex in theoretical basics or difficult to implement $[5,6]$. On the other hand, a PID controller offers one of the simplest ways to control a DC motor [7].

\section{BACKGROUND OF THE STUDY}

It is necessary to improve upon the initial DC Motor control systems to ensure the stability and efficiency of any given system. There have been studies concerning the way a DC motor is controlled yet the most popular is the method where a PID controller is utilized [8]. However, the quality of control systems, in general, suffer due to the high nonlinearity of the DC Motor [9,10,11]. In existing studies on DC motor controllers, becomes very complex when implemented which leads the researchers to further investigate and enhance the design methodologies of PID controllers due to the DC controllers' undesired gain.

DC motors can sometimes fail, and these failures tend to become difficult to overcome especially low resistance failures. Low resistance is caused by the degradation of the insulation of the windings, as a motor or any machine for that matter, moves it will most likely cause friction. Friction can wear down other elements in a system such as insulations. This can be due to overheating, due to the friction from moving parts, corrosion which is due to an element's exposure to oxygen and moisture, or other physical damages [12]. An enhanced control system will ensure that the system is efficient, and the desired output is obtained. Due to the simplicity of the PID controller, it has been commonly used in the industry. It has been studied that multiplexers can be used as a switching mechanism for the gain parameters, it has been observed that a reconfiguration occurs in every stage of the execution cycle. These PID controllers have been observed to have powerful computational and demand for storage due to its requirement of several algebraic operations which include matrix systems [13]. 


\section{STATEMENT OF THE PROBLEM}

DC motors are often used in industries using speed control systems for achieving the desired output. DC motors are often controlled manually by varying the armature resistance. Here, DC motor speed is changed through variation of the current in armature using a variable resistor or a rheostat. However, this method is not efficient since it takes us more time for adjusting the speed from its minimum and maximum rotations per minute. Another way of driving the DC motor is through the magnetic flux control method. Here, the flux is varied through the amount of current passing through the field winding. These methods of controlling a DC motor take up more time and are costly. Thus, using a digital controller for driving a DC motor should be easier to implement and analyze, as well as reduce the costs.

\section{SIGNIFICANCE OF THE STUDIES}

DC motors are very popular in industrial applications. DC motors are commonly used in speed control applications and systems concerning servos. The common components of a servo system consist of a motor, a device responsible for feedback or feedforward depending on the design, and a controller. The control system typically consists of a motion controller, meaning this control system is responsible for the motion profile of the motor and also provides the motor drive which is also the supply of power for the motor. On the other hand, PID controllers or Proportional Integral Derivative controllers are the most commonly used when it comes to controlling the DC motor due to its ruggedness, easy tuning and ease of implementation. The controlling system will be enhanced if proper metaheuristic techniques are used.

Metaheuristic techniques are used to optimize the PID controller thus optimizing the control system for the DC motor. In this paper, the metaheuristic technique used is a hybrid SA-NM, it has an objective function that contains the exponential factor and also the SA algorithm which is guided by the Boltzmann distribution of the energies with different states in an exponential function.

\section{DESCRIPTION OF THE SYSTEM}

The system consists of an input desired speed which shall be the setpoint, the input of a digital PID controller will be connected to the error calculated $\mathrm{e}(\mathrm{t})$ from the setpoint and feedback loop which was drawn from the actual speed of the motor and an introduced noise. Figures 1 and 2 shows the DC motor diagrams.

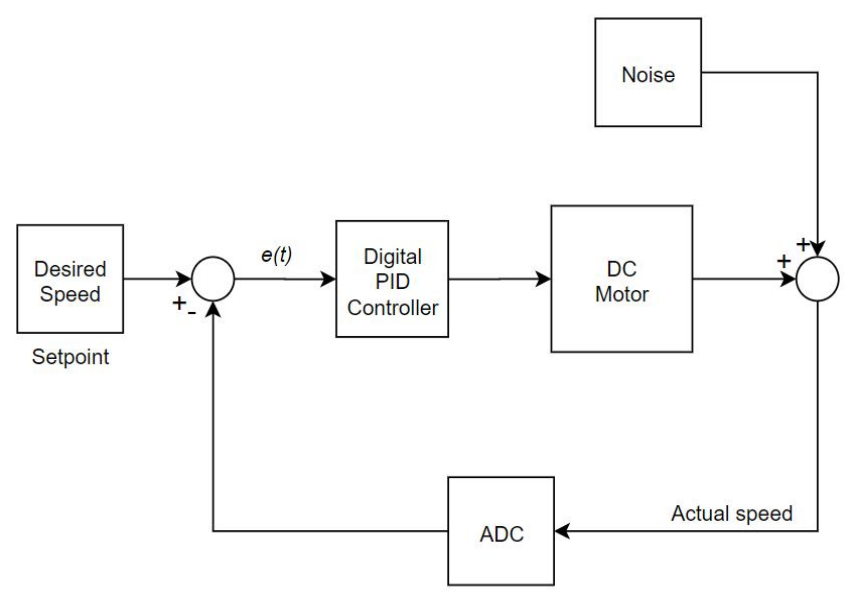

Figure 1:Block diagram of the system

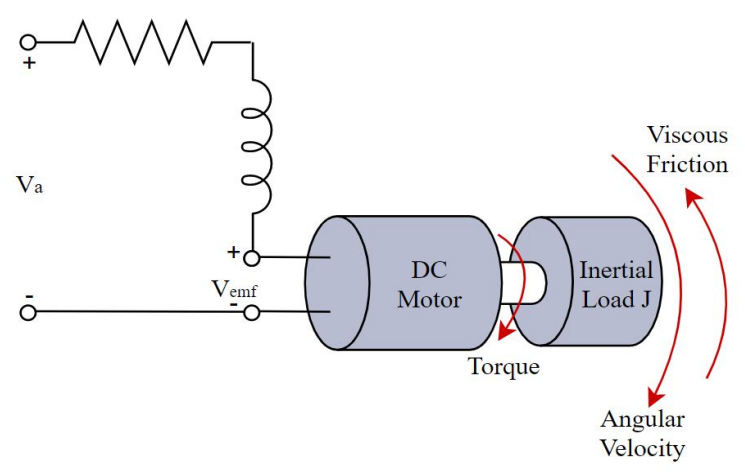

Figure 2:Diagram of the DC motor

\section{METHODOLOGY}

Figures 3 to 9 shows the Control System components.

\subsection{Components}

\section{A. DC Motor}

A DC Motor converts electrical energy into mechanical energy. Shown in figure 3 is the circuit diagram of a DC motor. The parameters which define the motor include the resistance, inductance, inertial load and back emf.

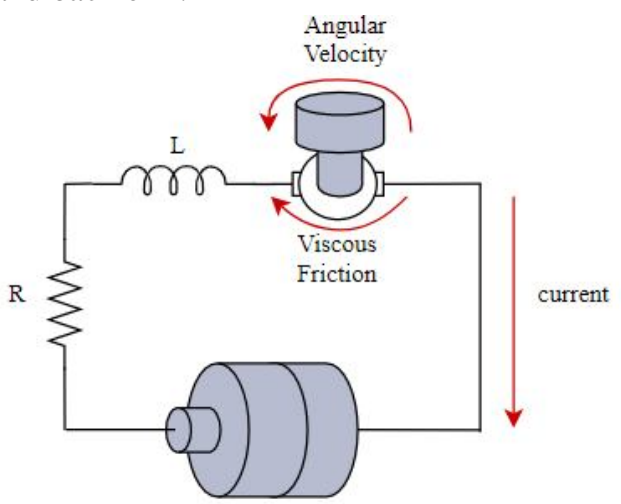

Figure 3: DC Motor Circuit 
B. Discrete PID controller

The discrete PID controller block is shown in figure 4. This control will be the one to compute for the error signal of the speed and voltage requirement to drive the motor.

\section{$\operatorname{PID}(z)$ Discrete PID Controller.}

Figure 4: PID Controller Block

C. Analog-to-Digital Converter

Since the control system is digital, analog signals need to be converted to digital signals. This is done using the analog-to-digital converter.

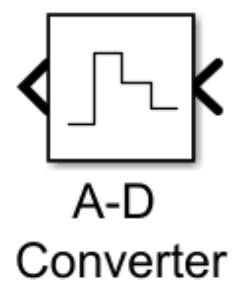

Figure 5: Analog-to-Digital Converter Block

D. Inputs/Outputs

1. Torque Disturbance

The torque disturbance is one external factor that affects the DC motor. The disturbance block is shown in figure 6.
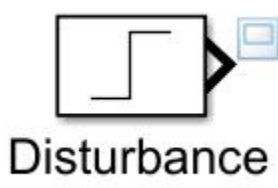

Figure 6: Disturbance Block

2. Desired Speed

For digital systems, a user can input its desired speed and the controller will be the one to compute the amount of voltage to be sent for that DC motor to have that speed.

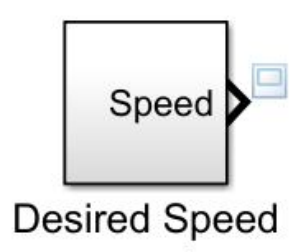

Figure 7: Speed Block
3. Sensor Noise

All motors are prone to noise and this block will Gaussian distributed random number as the noise.



Figure 8: Noise Block

\subsection{Simulation}

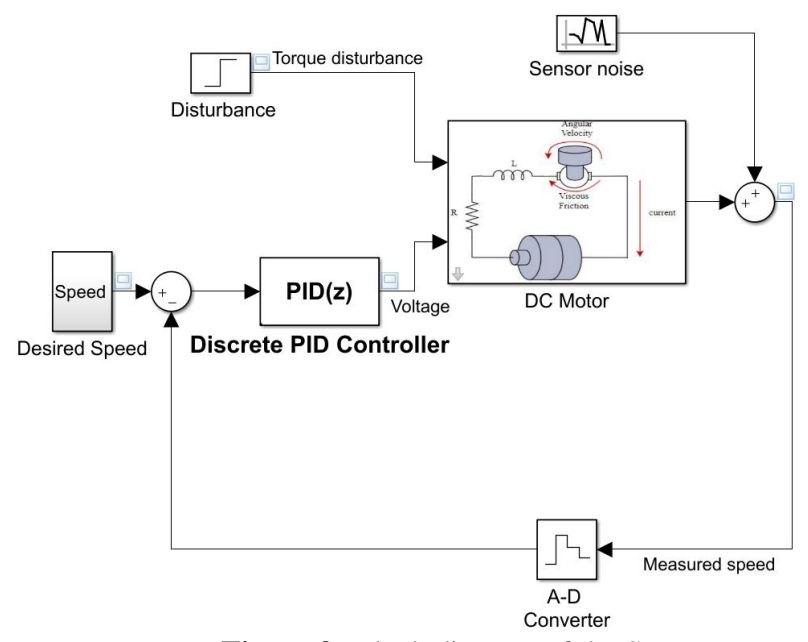

Figure 9: Block diagram of the System

\subsection{Procedure}

A. Open Simulink.

B. Place components and connect based on the block diagram given.

C. Speed Block.

1. Double click on the speed block.

2. Double click on the step block. This will provide a step output for the speed.

3. Set the desired values for each parameter.

4. Click "Apply" to apply the new values entered.

5. Click "OK"

D. Disturbance Block

1. Double click on the disturbance block.

2. Double click on the step block. This will provide a step output for the torque disturbance.

3. Set the desired values for each parameter.

4. Click "Apply" to apply the new values entered.

5. Click "OK"

E. Sensor Noise

1. Double click on the sensor noise block.

2. Double click on the step block. This will provide a Gaussian distributed random number output as the step noise. 
Aaron Don M. Africa et al., International Journal of Emerging Trends in Engineering Research, 8(9), September 2020, 5555 - 5562

3. Set the desired values for each parameter.

4. Click "Apply" to apply the new values entered.

5. Click "OK"

F. A-D Converter

1. Double click on the analog-to-digital converter block.

2. Input the sampling time of the converter;

3. Click "Apply" to apply the new values entered.

4. Click "OK"

G. PID Controller

1. Double click on the Discrete-Time PID Controller block.

2. Make sure that the controller is set to PID and time domain discrete time.

3. Set the parameters for the PID controller and input the gains on the boxes.

4. Set the sampling time the same as the sampling time in the analog-to-digital converter.

H. Run the simulation. The result of the entered parameters will be displayed. It will show if the digital control system is performing well through the waveforms of the desired speed versus the measured speed of the motor.

I. Stop the simulation. Make the necessary adjustments in the parameters of the discrete-time PID controller. If the gains have been calculated, it may be set there. If not, the system may be tuned.

1. Tuning the controller.

a. Double click on the discrete-time PID controller.

b. Click on the "Tune" button. The PID tuner of the Simulink Control Design will open.

c. The PID tuner will tune the system by linearizing the plant and computing for the gains. A graphical user interface (GUI) will then open and show the step response of the block having the current parameter values in comparison to the tuning done.

J. After tuning, run the simulation again. The performance of the digital control system will be shown.

K. The response time of the controller may be adjusted for a faster response or a smaller overshoot.

\section{REVIEW OF RELATED LITERATURE}

\subsection{Motors}

As one of the traditional machines, DC motors have been widely used since the 19th century, due to its high torque and low volume [14]. In industries, there are two main types of DC motors: the conventional DC motor and the brushless DC
(BLDC) motor. The first type of DC motor, the conventional one, the flux is produced by the current through the field coil of the pole structure. While the latter has permanent magnets that provide the essential air gap flux instead of poles. In DC motors, it has been observed that additional parameter changes or sudden loa variations changes the behavior of the whole system in such a way that the system obtains new oscillations, that is why in this paper, it was concluded that the mechanical and electrical constraints of DC motor systems with PID controllers must be verified as constraints before implementation.

In comparison to the other electric machine, such as induction machines [15,16], DC motors have some internal advantages. It has the advantage of simple control and a large electromagnetic torque. Not to mention its ability to adjust its speed in wide ranges [17]. Controlling the DC motors have been continuously studied up to this day. There are many published studies to control DC motors, such as nonlinear control [18], optimal control [19], and adaptive control [20]. These controls are also applied to diverse types of DC motors including BLDC. Brushless DC motors have become more successful in the industries, especially in the fields of production, aeronautics, robotics, computer peripherals, industrial automations, etc [21]. However, due to complexity of the implementation of these control methodologies [22], the conventional PID controllers were used to be able to get the job done.

\subsection{PID Controller}

PID controllers have covered treatment for transient and steady-state response to give the simplest and most efficient way to solve many real world control problems. With this, the PID controllers are the most widely used control strategy up to today. It is estimated that over $90 \%$ of control systems employ PID control, due to the derivative gain set to zero (PI control). The PID controller has three terms which fulfills three common requirements of most control problems. It has an integral term that yields a zero steady-state error in tracking a constant setpoint. This integral control also enables the complete rejection of constant disturbances. While the integral term controls and filters the higher frequency sensor noise, the proportional term responds immediately to the current error. However, these two terms have their own disadvantages. The integral term is slow in response to the current error, while on the other hand, the proportional terms cannot achieve the setpoint accuracy without an intolerably large gain [23].

Despite the simplicity of the PID controller and its high efficiency, the tuning gains of this controller have been difficult; thus, researchers tend to enhance the design methodologies of the existing PID controllers. The control structure has organized the convex set with an internal PD 
Aaron Don M. Africa et al., International Journal of Emerging Trends in Engineering Research, 8(9), September 2020, 5555 - 5562

controller, between the PID controller and PI controller. Researchers have been studying and comparing the results of PID controllers to Fuzzy-PID controllers. This has been an interest since conventional PID controllers still leave the difficulty of controlling the value of parameters and get the characteristics suitable to each parameter. While on the other hand, fuzzy controllers satisfy the control characteristics and provide easy computing, to be able to control the motor efficiently. Before implementation of a PID controller to a DC motor control system, critical gains should be calculated to ensure the stability of the system as a whole. With the proper installation of a PID controller, it can ensure protection against large steady state RMS, steers, frequency and also provide protection behaviors were unforeseen.

\section{THEORETICAL CONSIDERATIONS}

Tuning PID controllers is essential for proper operation, because an improperly tuned controller can lead to unnecessary operational and maintenance costs in industrial applications. There are three basic parameters in a PID controller i.e. the proportional, integral, and derivative. Proportional controllers can modulate the output of a system depending on its setpoint; however, it cannot eliminate errors from the output or process variable. An integral part of the controller can help eliminate this error. Furthermore, adding the derivative component can help increase the responsiveness of the system. Improving a PID controller can be done either at a hardware-level or a software/algorithm-level. Going through either route is an investment with risks and rewards depending on the implementation. This paper mostly discusses tuning the PID gains of the controller using a tuner with a graphical user interface (GUI) in MATLAB. In systems where a PID controller is utilized, a commonly used technique to test and analyze the performance of the PID controller is to subject the motor to the most common load variations a motor will encounter and also changing the load variations gradually under constant speed control.

\section{DATA AND RESULTS}

Figures 10 to 21 shows the simulation output.

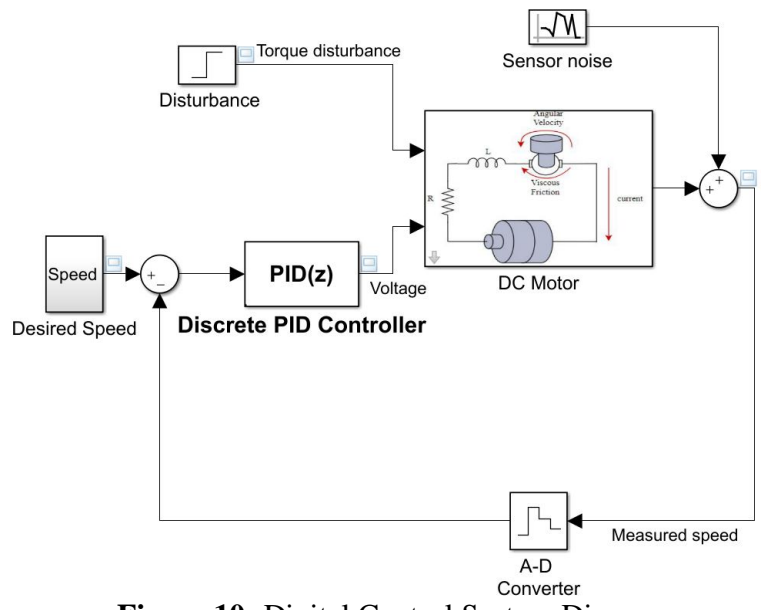

Figure 10: Digital Control System Diagram

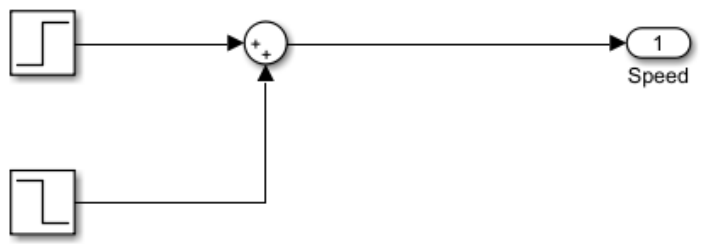

Figure 11: Diagram of the Speed Block

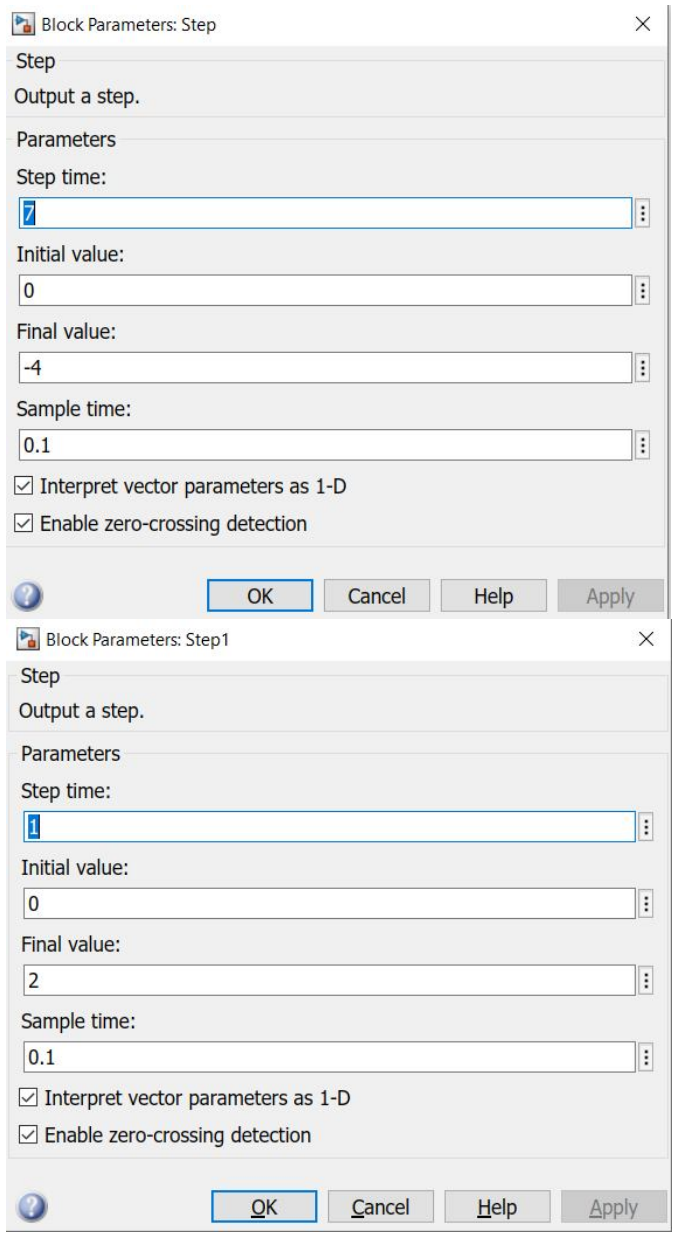

Figure 12: Initial Parameters of the Step for the Speed

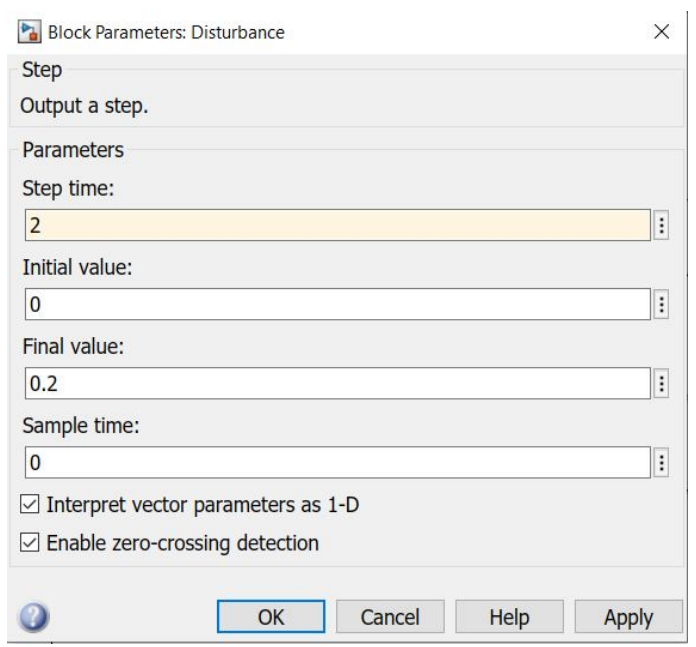

Figure 13: Initial Parameters of the Disturbance Block 
Aaron Don M. Africa et al., International Journal of Emerging Trends in Engineering Research, 8(9), September 2020, 5555 - 5562

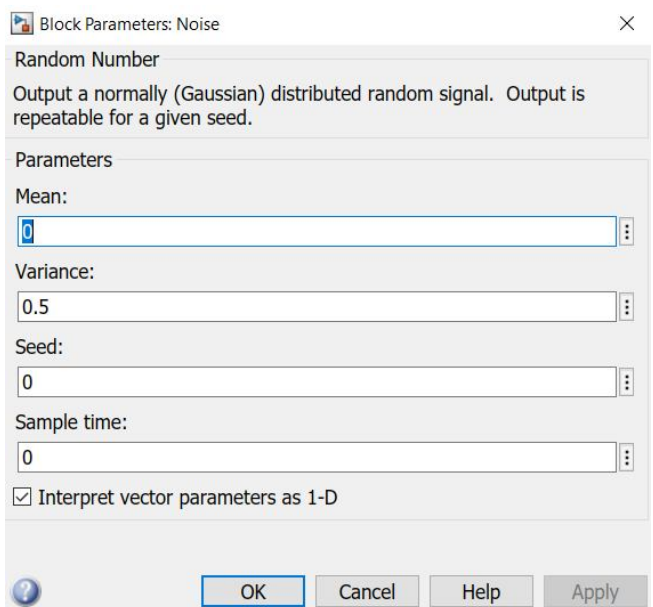

Figure 14: Initial Parameters of the Noise Block

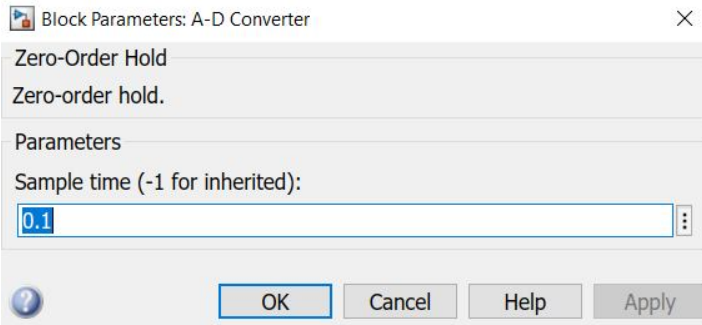

Figure 15: Initial Parameters of the Analog-to-Digital Converter



Figure 16: Initial Parameters of the Discrete-Time PID Controller

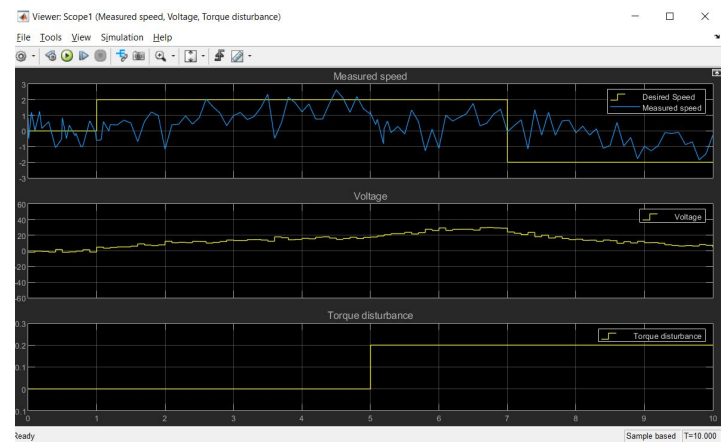

Figure 17: Desired Speed vs. Actual Speed Performance



Figure 18: PID Tuner GUI

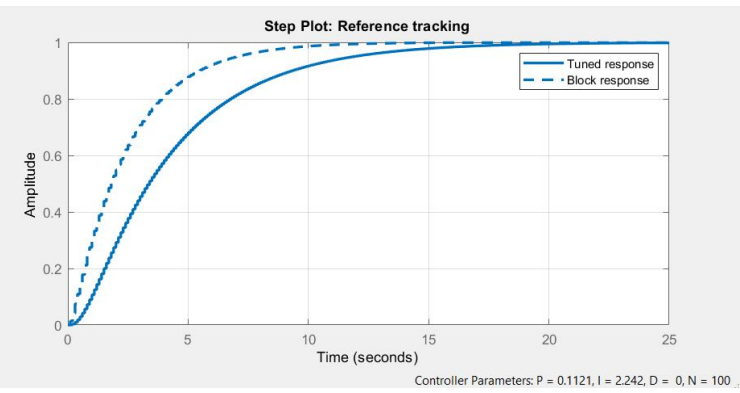

Figure 19: Current Step Response of the Controller vs. Tuned Step Response

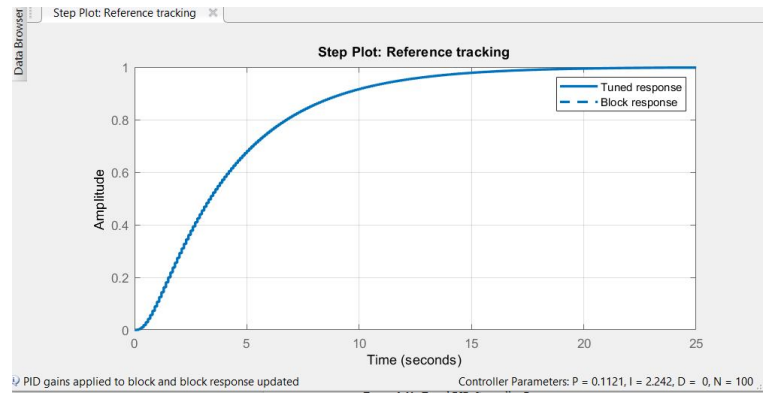

Figure 20: Updated Block Response with Calculated Gains Applied



Figure 21: Updated Gains on the Parameters of the PID Controller 


\section{ANALYSIS OF DATA}

The graphs show the performance of the measured speed which is the blue line in comparison to the desired speed which is the yellow line. As seen, the measured speed tracking is not doing well due to the noises introduced to the system and the gains of the discrete-time controller. The voltage sent to the motor is shown in the second graph. In order to improve the overall performance of the system, it has been tuned using the PID tuner of the Simulink Control System Toolbox. Tuning the PID controller will linearize the plant and compute for the gain needed of the controller. The tuning tools are on the top and can be used for changing the response time of the block. The calculated gains are now set in the parameters of the block.

\section{CONCLUSION}

In conclusion, the group was able to successfully develop and tune a PID-based DC motor control system. The PID controller and DC motor were both simulated using Simulink and the Control Systems Toolbox in MATLAB. After successfully simulating the system, tuning was done to improve the performance of the PID controller and overall, the system. Adjusting the step response of the controller also led to the gains of the controller changing. These two parameters were key in adjusting the performance of the system. Depending on the configuration, one could achieve either faster response time or better stability.

Without tuning, the DC motor was very unstable, and its speed was very erratic. The entire digital control system was created for controlling the speed of the DC motor by calculating the error between the measured speed and the desired speed then sending the voltage to the motor. It is also important to include the noise since the motor is prone to external noise. This noise was combined with the output of the motor. Since the signals are analog, the analog-to-digital converter is essential to convert the signal to digital before being combined with the desired speed.

After tuning the system by adjusting the gain using the PID tuner, the system performed noticeably better. Not only did the output speed get closer to the setpoint, but the step response of the system also got faster. It is important to note, however, that the increased step response came at the cost of system stability as the faster system response caused more overshoot and noise. This can be remedied by lowering the step response of the system.

\section{RECOMMENDATIONS}

Further improvements can still be made to this project as there are a number of ways to design a DC motor control system. One such innovation is the possible addition of a fuzzy logic algorithm to the design. The addition of a fuzzy logic controller should add another degree of complexity; however it could have the capacity to improve on complex and non-linear processes. Theoretically, in the presence of load and possible noise the addition of a fuzzy logic control should compensate for the errors better than a PID controller on its own. Furthermore, comparing the performance of a PID controller with a fuzzy logic enabled one with regards to the speed, position, and torque would give a better understanding of how they differ. Determining the practicality of implementing a fuzzy logic controller on a PID controller would be recommended, because the added benefits of using fuzzy logic would come at a cost be it financially or timely.

Additionally, there are different methods of tuning a PID controller. It is recommended that more methods of PID tuning should be studied. This is because different methods could yield different results and better implementation both economically and in practicality. Similar to fuzzy logic, another innovation in the field of PID controllers is called the Adaptive PID controller. In this implementation, the design revolves around the error between the desired output and process response to zero. It is based on the model reference adaptive control method.

A comparison between the Adaptive PID controller and the Fuzzy logic enabled PID controller is an ideal study as these two are the most prominent innovations with respect to PID control systems. In addition to that, PID controllers are not limited to DC motors. They can be applied to many different industrial applications such as thermostats, control valves, and power converters.

\section{REFERENCES}

[1] N. Shiehand P.Tung, "Robust output tracking control of a linear DC brushless Motor for transportation in manufacturing system," IEE Proceedings-Electric Power Applications, pp. 119-124, 2001.

[2] A. Africa, M. Esteban and H. Orines, "Vehicular speed limiting control system in critical zones using global positioning system," International Journal of Emerging Trends in Engineering Research, vol. 8, no. 5, pp. 1916-1919, 2020.

[3] F. Hidayanti, H. Santoso, E. Wati and G. Prasetyo, "Design of miniature complex loop input/output simulator for fuel gas and seal gas filter separators by using distributed control system," International Journal of Emerging Trends in Engineering Research, vol. 8, no. 7, pp. 3122-3128, 2020.

[4] T. Trong, "The Control Structure for DC Motor based on the Flatness Control," International Journal of Power Electronics and Drive Systems (IJPEDS), vol. 8, pp. 1814-1821, 2017.

[5] H. Ibrahim, F. Hassan, and A. Shomer, "Optimal PID control of a brushless DC motor using PSO and BF techniques," Ain Shams Engineering Journal, vol. 5, no. 2, pp. 391-398, 2014.

[6] C.Lin and H.Jan, "Evolutionarily multiobjective PID control for linear brushless DC motor," in Proc. of IEEE Int. Conf. Industrial Elect. Society, vol. 3, pp. 2033-2038, 2002. 
Aaron Don M. Africa et al., International Journal of Emerging Trends in Engineering Research, 8(9), September 2020, 5555 - 5562

[7] K. Ang, G. Chong and Y. Li, "PID control system analysis, design, and technology," IEEE Trans Control Syst Technol 2005, pp. 559-76.

[8] M. Bosco, J. Guedes, M. Castoldi, A. Goedtel, E. Silva, and L. Buzachero, "Estimation of parameters and tuning of a speed PI of permanent magnet DC motor using differential evolution," 2017 IEEE International Electric Machines and Drives Conference (IEMDC), 2017.

[9] J. Yao, Z. Jiao, and D. Ma, "Adaptive Robust Control of DC Motors With Extended State Observer," IEEE Transactions on Industrial Electronics, vol. 61, no. 7, pp. 3630-3637.

[10]D. Xue, C. Zhao, and Y. Chen, "Fractional order PID control of a DC-motor with elastic shaft: A case study," 2006 American Control Conference.

[11] N. Thomas and P. Poongodi, "Position control of DC Motor using genetic algorithm based PID controller," Proceedings of the World Congress on Engineering, vol. 2, 2009.

[12] M. Sabir and J. Ali Khan, "Optimal Design of PID Controller for the Speed Control of DC Motor by Using Metaheuristic Techniques," Advances in Artificial Neural Systems, 2014.

[13] S. Khan and K. Papadimitriou and Buttazzo, Giorgio andKalaitzakis, "A Reconfigurable PID Controller," 2018.

[14] D. Somwanshi, M. Bundele, G. Kumar, and G. Parashar, "Comparison of Fuzzy-PID and PID Controller for Speed Control of DC Motor using LabVIEW," Procedia Computer Science, vol. 152, pp. 252-260, 2019.

[15] B. Abdelhak and B.Bachir,"A High gain observer based sensorless nonlinear control of induction machine," International Journal of Power Electronics and Drive Systems, vol. 5, no. 3, p. 305, 2015.

[16] R. Gunabalan and V. Subbiah, "Speed Sensorless Vector Control of Induction Motor Drive with PI and Fuzzy Controller," International Journal of Power Electronics and Drive Systems, vol. 5, no. 3, p. 315, 2015.

[17] K. Ang, G. Chong, and Y. Li, "PID control system analysis, design, and technology," IEEE Trans Control Syst Technol 2005, pp. 559-76, 2005.

[18] N. Hemati, J. Thorp, and M. Leu, "Robust nonlinear control of Brushless de motors for direct-drive robotic applications," IEEE Trans. Ind. Electron., vol. 37, pp. 460-468, Dec 1990.

[19] P. Pelczewski and U. Kunz, "The optimal control of a constrained drive system with brushless dc motor," IEEE Trans. Ind. Electron., vol. 37, pp. 342-348, 1990.

[20] E. Cerruto, A. Consoli, A.Raciti,and A. Testa, "A robust adaptive controller for PM motor drives in robotic applications," IEEE Trans.Power Electron., vol. 10, pp. 62-71, 1995.

[21] C. Kim, J. Yang, D. Lim, and B. Suh, “An Enhanced PID Controller for Speed Control of Brushless DC Motors Based on Convex Set Optimization," IFAC Proceedings vol. 43, no. 22, pp. 75-80, 2010.

[22] C. Lin and H. Jan, "Multiobjective PID control for linear brushless DC motor: an evolutionary approach," Proc.
IEEE Int. Conf .Industrial Elect.Society, vol. 149, no. 6, pp. 397-406, 2002.

[23] C. Knospe, "PID control. Control Systems Magazine," IEEE, vol. 26. Pp. 30-31, 2006. 